\title{
Probing Into Space-Time View
}

\author{
Chang-Wei Hu \\ Beijing Relativity Theory Research Federation, Beijing, China \\ Shanghai Senior Scientist and technician Association, Shanghai, China
}

\begin{abstract}
Space-time view is the cornerstone of physics, which can be divided into two categories. One is the absolute space-time view, it is a description of pure and real space-time. Because there is no a space with nothing, the absolute space-time view will deviate from the actual measured value quantitatively. The second is the view of material space-time, which is the result of observing the world through some medium. The principles of cosmology play an important role in the view of material space-time. The relativistic space-time view is a material space-time view, its material basis is the physical vacuum, that is, ether. The invariability principle of light velocity is intrinsically related to the principle of cosmology.
\end{abstract}

Key words: Space-time theory, Cosmological principle, Theory of relativity, Medium of space-time, Physical vacuum (Ether)

\section{Introduction}

Space and time are the most basic physical quantities, and space-time view is the most basic points of view of physics.

S. Hawking and L. Mlodinow said: "The goldfish is seeing a version of reality that is different from ours, but can we be sure it is any less real? For all we know, we, too, may spend our entire lives staring out at the world through a distorting lens. In physics, the question is not academic. Indeed, physicists and cosmologists are finding themselves in a similar predicament to the goldfish's......each describing its own version of reality, as if it viewed the universe through its own fishbowl."[1] They considered that the goldfish's version of reality is different from ours, therefore if goldfish are intellective, they can establish a theory of physics that is different from ours. How is the physical theory of intelligent fish? They have not research further. This question is quite interesting and meaningful. We find that if intelligent fish take water as their living space and apply the cosmological principles, they can get a space-time theory similar to relativity. This means that just as the fish are seeing the world through the water, the theory of relativity are describing world through a special medium, which has great enlightenment action to further understand the theory of relativity and even entire modern physics.

The space-time view of intelligent goldfish can be divided into two categories, one is the spacetime view that goldfish describes the world outside the fishbowls; the second is space-time view that the goldfish describes the world within the fishbowls.

Looking at the world with light, the scene that goldfish see the view outside the fishbowls is different from ours, because glass and water can reflect a light. Obviously, the space-time view outside the fishbowls of the intelligent goldfish would vary with the material and shape of the fishbowls, which is difficult to be described unitedly, so that it is of little research value. The spacetime view within fishbowls of intelligent fish is only related to water, and most fish live in ocean, such a space-time view has certain universality. Let's start with that.

\section{The space-time view of intelligent fish}




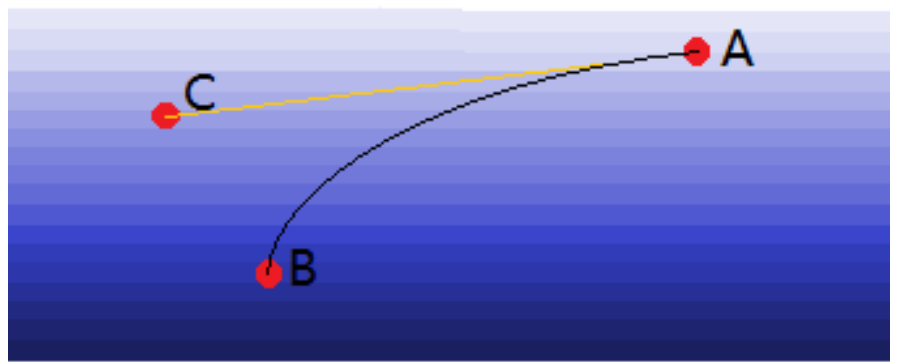

Figure 1. The space-time theory of wise fish within fishbowls.

As we know, the density of seawater can vary with factors such as depth, temperature, etc. Inhomogeneous medium is a graded media, where light or sound will bend in the direction of greater density. As shown in Figure 1, the size of the water density is indicated by the shade of blue, and the path of the sound or light emitted by fish B to fish A is curved (of course, the bend is actually very small), so fish $A$ will mistakenly consider that fish $B$ is at point $C$; if fish $A$ swims, point $C$ would vary with it. Therefore the space-time view of intelligent fish will be local, and it is very different from our daily Newtonian space-time view. So, how will the wise fish derive the mathematical expressions of their space-time view? Let's explore it.

The uniformity of space is a cosmological principle established in both the absolute and relativistic space-time theories, and it should also be established in the fish's space-time theory. Water is the living space of fish, they can assume that in quantitative description, the distribution of water is uniform everywhere and isotropic. This means that the density of water can't change, or that water is incompressible. That is to say, in the absolute space-time theory, water is compressible, while in the space-time theory of intelligent fish, water is uncompressible. According to this clue, it is possible to obtain the mathematical expression of the wise fish's space-time theory.

In fluid mechanics, the mathematical formula for converting compressible fluid $\varphi(\mathrm{x}, \mathrm{y}, \mathrm{z})$ into incompressible fluids $\varphi\left(\mathrm{x}^{\prime}, \mathrm{y}^{\prime}, \mathrm{z}^{\prime}\right)$ is [2]:

$$
x^{\prime}=\beta x \quad y^{\prime}=y \quad z^{\prime}=z \quad\left(\beta=\frac{1}{\sqrt{1-v^{2} / c^{2}}}\right)
$$

Where the $\mathrm{v}$ is velocity, $\mathrm{c}$ is sound speed in this fluid.

Equation (1) converts a compressible fluid into an incompressible fluid, and also transforms the expression of absolute space-time theory into the expression of space-time theory of a wise fish.

Substitute (1) into the Galileo transformation which expresses the absolute time-space view (2), (3):

$$
\begin{array}{lll}
x_{2}=x_{1}-v t_{1} & y_{2}=y_{1} & z_{2}=z_{1} \\
x_{1}=x_{2}+v t_{2} & y_{1}=y_{2} & z_{1}=z_{2}
\end{array}
$$

It can derive the expression of space-time view of wise fish. Note: The $t_{1}=t_{2}$ in the Galileo transformation no longer is applicable, and to derive the relationship between $t_{1}$ and $t_{2}$ should substitute (1) into simultaneously (2) and (3). The result is:

$$
x_{2}^{\prime}=\beta\left(x_{1}^{\prime}-v t_{1}\right) \quad y_{2}^{\prime}=y_{1}^{\prime} \quad z_{2}^{\prime}=z_{1}^{\prime} \quad t_{2}=\beta\left(t_{1}-v x_{1}^{\prime} / c^{2}\right)
$$

Eq. (4) is the expression of space-time view of wise fish. If $c$ is the speed of light in a vacuum, (4) is the Lorenz transformation! In fact, this also is the process that we derived the Lorenz transformation with the method of fluid mechanics [3].

Wise fish regard water as living space, and their space-time is a material space-time. Its mathematical expression is similar to the relativistic space-time theory. Does this mean that 
relativity is also a material space-time? Yes.

\section{Two space-time theories with different properties}

In fact, the correlation between fluid mechanics and Lorenz transformations has long been recognized. In the 1980s, Liao, Mingsheng pointed out that similar to relativistic equations can be derived from the fluid mechanics [4], and later he proposed 'fluid invariant theory' [5]. Yang, Xin Tie and others further explore the intrinsic relationship between fluid mechanics and the principle of general covariate and general relativity line elements $[6,7]$.

The author once pointed out in the 1980s that light is the second sound in ether, and Lorenz transformation can be derived by the method of fluid mechanics [8]. Later, on the basis of this view, "the theory of compressibility ether" was established [3,9].

Vacuum is not empty, any space will be filled with matter [10]. Ether is generally called a physical vacuum, a kind of vacuum-state matter. The space-time theory what Lorenz transformation represents is a material space-time theory, in which the speed of light c, is the speed of sound in the ether. Here Hawking's conjecture is confirmed: "We, too, may spend our entire lives staring out at the world through a distorting lens." Yes, just as fish see the world through water, mankind see the world through ether. The relativistic effects are the lens effects of ether. In the case of low-speed, weak gravitational field, ether lens is like a flat-light lens, its action is insignificant, and absolute space-time theory can be established. In the case of high-speed, strong gravitational field, ether lens becomes a concave-convex lens, its action is obvious, thus relativistic effects occur.

The medium that as material basis of space-time view, we call it space-time medium. It is water in the space-time view of wise fish; it is ether in relativistic space-time view.

In the past, it was thought that the space-time of physics was the same, Newton's absolute space-time theory was an approximate description of this space-time, and the relativistic space-time theory was an accurate description of it. It is wrong to think so. Absolute space-time has nothing to do with any matter, and relativistic space-time is closely related to ether.

The mathematical expression of absolute space-time view is Galileo transformation, in which the time interval and space interval are the invariant, namely the standards of time and space is absolutely unchanged, which is the most basic nature of absolute space-time view. Absolute time and space have nothing to do with matter, are not affected by the environment, and are truly spacetime. Because of the invariance of space-time standards, we can establish a rigid three-dimensional coordinate system and a one-dimensional timeline anywhere, which is a measurable mathematical description of space and time in the absolute space-time view.

In the special relativity, the coordinate axes and timelines are not rigid, They will expand and contract with the speed of movement, which shows that the standard of space-time are variable, and space and time are no longer independent of each other, they has been mixed into a four-dimensional geometric continuum, but its four-dimensional space-time interval is invariant, can still be regarded as Euclidean straight space. Lorenz transformation is a mathematical expression of this space-time view.

In the absolute space-time view, ether is a kind of compressible fluid, whose density is variable; while in the relativistic space-time theory, ether is incompressible and its density is constant. This means that the relativistic space-time standard can vary with ether density in absolute space-time: the unit length is proportional to the interval of the adjacent particles of the ether, and the unit time is proportional to the time interval that light passing through the interval of adjacent ether particles.

The theory of compressibility ether considers that the ether density in the absolute space-time 
view corresponds to the absolute value of the gravitational potential in quantitative description. The special and general relativity effects, which look very different, in fact, both of them can be attributed to the change of ether density, because physical quantities such as length, time and mass all can vary with ether density. The general relativistic effect is that the physical quantity vary with gravitational potential; while the kinematic effect of special relativity is that when the object moves in the compressible ether, the ether density where it goes will be higher than when it is stationary. As for the relationship between gravitational potential and velocity, we can derive a simple expression by the equivalence principle and the law of energy conservation: Let an object falls free towards an isolated gravitational field of a heavenly body, its initial velocity is zero; when it is $r$ away from the heavenly body, the velocity is $\mathrm{v}$ and the gravitational potential is $\varphi$, (which is zero where it is infinite away from the heavenly body), its sum of potential energy and kinetic energy are always equal to zero: $\frac{1}{2} m v^{2}+m \varphi=0$, namely:

$$
|\varphi|=\frac{1}{2} v^{2}
$$

In addition, general relativity further regards the rate of change of ether density as the curvature of relativistic space-time, so it regards the straight absolute space-time (through the ether) as curved relativistic space-time.

Where the absolute value of the gravitational potential is bigger, the clock walks slower. The value of the gravitational potential is determined by two factors, one is the gravitational field, which is $\mathrm{GM} / \mathrm{r}$, where $\mathrm{G}$ is the gravitational constant, $\mathrm{M}$ is the mass of the object, $r$ is the distance from the center of mass; the second is speed, which is expressed by Eq. (5). Imagine an artificial planet run along Earth's orbit. The clock on this artificial planet and the clock on the ground, which one is slower? Relative to the sun, the two speeds around the sun is the same, and the clock on the ground has an extra speed of the earth's rotation; their solar gravitational potential is the same, and the clock on the ground has an extra earth's gravitational potential. Thus the clock on the ground walks slower. This results are contrary to the general view that astronauts will be younger and should be tested by designing experiments. Of course, in the initial stages of launch, the clock on the artificial planet goes slower than the clock on the ground.

Macroscopic ether is an invisible fluid. In the absolute space-time view, ether density would fluctuates with the gravitational potential, which looks simple. However, in people's impression, ether (physical vacuum) is a very complex thing, how is this going on? Here's the thing: People is in the microscopic system to know that the vacuum is not empty, and think that the ether is the ground state of quantum field. The composition of quantum fields is varied, which creates the complexity of microscopic ether. Our view is: there are many interval field ethers in nature, and humans only know one of them - gravitational field ether; so that when we look at gravitational field interval (macro-world), the things are quite intuitive and clear; when we observe micro-world, things look strange and fuzzy [11]. Specifically, the micro-ether "lens" is more "rough" than the macro. In the relativistic space-time view, the ether is evenly distributed, and the rough micro-ether "lens" is like an evenly arranged array of microlens. F Wilczek once said that vacuum is a kind of "grid"[12]. Yes, innumerable microlenses constitutes what Wilczek calls a vacuum "grid". Microscopic particles travel through such a vacuum, and there are many possibilities at every step, and path integrals are a true description of this.

Modern physics tells us that the space-time of the high-speed state is different from that of the 
low-speed state; the micro world is even more different from the everyday world. We point out that the real space-time is invariable, what distorts the image of things is the role of the ether and its microstructure. We can use the action of ether to explain the physical mechanism of modern physics on the basis of absolute space view.

\section{The important action of cosmology principles in the view of material space-time}

The derivation of Lorenz transformation is inseparable from the cosmological principle [13]. The derivation process of the wise fish's space-time view is: the living space (water) of the fish + the cosmological principle $\rightarrow$ the fish's space-time view. As in the case of relativity, we can speculate that in the space-time theory of the wise fish, due to the cosmological principle, the density distribution of water is uniform everywhere, i.e. the interval of the adjacent particles of water is equal, and the unit length is proportional to the interval of the adjacent particles of the water, and the unit time is proportional to the time interval that sound passing through the interval of adjacent particles of water, which means that the cosmological principle play a role in determining quantitative standards.

The basic meaning of the cosmological principle is: the uniformity of space and the isotropic. In the absolute time-space view, the absolute invariance of space-time standards leads to the uniformity of space and isotropic. Here the cosmology principle is a result. In the material spacetime view, the cosmology principle is a prerequisite.

In relativity, the quantitative criteria are determined by the invariability principle of light velocity, which means that the distribution of ether density is uniform everywhere and isotropic, therefore, the invariability principle of light velocity is the embodiment of the cosmological principle.

The cosmological principle has another a meaning: the distribution of matter in space is uniform and isotropic. This is not true in the microscopic and macroscopic systems, because there is universally agglomeration of matter: particle clusters integrated atoms, atomic clusters integrated molecules, molecular clusters integrated objects, objects clusters integrated star, star clusters integrated galaxies, and so on. It is entirely possible that this series of agglomeration will continue to extend in the larger aspects of the universe. Therefore, it can only be a statistical approximation at best.

There are many continuous media in the real world. For example, air, human beings are inseparable from air, why do humans not use air as a space-time medium? This is mainly for two reasons: First, the constant $\mathrm{c}$ in the Lorenz transformation is a kind of limit speed, of all the speeds known to man, the light velocity is the fastest. In addition, the precision derivation of fluid mechanics of Lorenz transformation requires that the fluid is a full-space superfluid, the general fluid is difficult to satisfy, and the vacuum state ether can be fully satisfied.

The principle of cosmology is a quantitative convention to a space-time medium. It is this convention that leads relativistic quantitative effects [14], and relativistic quantitative relationships are only an appearance. For example, relativity shows that "the moving clock must be slow", there are A, B two people are relatively moving with speed v, A think B's clock is slower, and B think A's clock is slower, which clock is slower? It is difficult to confirm. It has been argued that relativistic effects are observational effects and are therefore only relevant to the observer. The theory of compressibility ether holds that the relativistic effects are the substantial effects of ether density change. The experiment of atomic clocks flying around the earth conducted by Hafele and Keating in 1971 showed that, on average, a flying clock is slower by $59 \times 10^{-9}$ seconds than a clock on the 
ground after flying towards the east, and the flying clock is faster by $273 \times 10^{-9}$ seconds than the clock on the ground after flying towards the west $[15,16]$. Obviously, in which the moving clock does not necessarily slow, and the results is not dependent on the observer.

In the space-time theory of intelligent fish, the sound speed of water is the extreme speed. But the wise fish will find that the speed of light is much faster than the speed of sound. So their spacetime theory will be upgraded to the relativistic space-time theory. Is it possible for the human space-time theory to escalate? According to the "multi-order hierarchical cosmological model"[11], it is entirely possible.

\section{Summary}

There are two kinds of space-time view, one is the absolute space-time view represented by Galileo transformation, which is the theory of real space-time that has nothing to do with any matter; and the other is the material space-time theory represented by the Lorenz transformation, where the constant $\mathrm{c}$ is not limited to the speed of light, is the speed of sound in a specific space-time medium, is set as constant by the cosmological principle, and as the quantitative reference of this space-time medium system.

Physics is an experimental science, and it must be quantitatively linked to actual measurements. The actual measurement is the comparison and action between two bodies. The absolute space-time view has nothing to do with matter, obviously it is difficult to fully match the actual measurements. The material space-time view can match the actual measurement within a certain range, but it distorts the real space-time, and its scope of application is limited. Therefore, we should grasp the physical mechanism of material space-time view on the basis of absolute space-time view.

\section{References}

1. Hawking, S. \& Mlodinow, L. The (elusive) theory of everything. Scientific American, October 69-71 (2010).

2. Fung, Y. C. A First Course in Continuum Mechanics, Beijing, Tsinghua University Press, pp. 257-259(2005).

3. Hu, C.-W. Derivation of the relativistic equations from classical continuum mechanics on the basis of a macroscopic vacuum. Physics essays, 27, 375-379 (2014).

https://doi.org/10.4006/0836-1398-27.3.375

4. Liao Ming-Sheng, The Founding of Flow Dynamics Relativity, Potential Science, 1989.4, pp.33-38

5. Liao Ming-Sheng, Invariability theory of fluid, Shanghai Science and Technology Publishing House, 1993.

6. Xintie Yang, Cunru Bai, Application of aerodynamic methods in the development of relativity, China Aerodynamics Conference Papers Collection, 2001-10.

7. Xintie Yang, Shuangren Zhao, The Principle of Covariant Invariability and the Metric Invariant of Extensional Relativity in Compressible Fluid, Beijing Radio University Journal (Nature Science), 2003

8. Gong Yue, The Ether Theory of Interval Field(in Chinese), Potential Science, 1989.4, pp.39-42.

9. Chang-Wei Hu, The theory of compressibility ether, Modern physics, 2017, 7(4): 112-133. https://doi.org/10.12677/mp.2017.74013。

10. Ye Yin, Multi-Layer Model of Matter Space on Universe, Journal of Jilin Normal University 
(Natural Science Edition), Feb. 2010, pp23-29.

11. Chang-Wei Hu, The Confusion of Modern Cosmology and the New Model, ScienceOpen Preprints, DOI: 10.14293/S2199-1006.1.SOR-.PPTYNQB.v1

12. Wilczek, F. Lightness of Being: Mass, Ether and Unification of Forces, Science News, 2009.

13. Chang-Wei $\mathrm{Hu}$, The logic structure deriving Lorentz transformation, ScienceOpen Preprints,2019.11.13。ID: 10.14293/S2199-1006.1.SOR-.PPBEOMV.v1

14. Hafele, C. and Keating, R. (1972) Around-the-World Atomic Clocks: Predicted Relativistic Time Gains. Science, 177, 166-167. https://doi.org/10.1126/science.177.4044.166

15. Hafele, C. and Keating, R. (1972) Around-the-World Atomic Clocks: Observed Relativistic Time Gains. Science, 177, 168-170. https://doi.org/10.1126/science.177.4044.168 [

16. Chang-Wei Hu. On the Quantitative Effects. International Journal of Modern Physics and Application. Vol. 1, No. 3, 2014, pp. 38-42. 